\title{
The rate of recovery in renal function when patients with HIV discontinue treatment with tenofovir
}

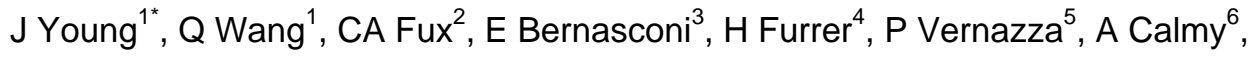 \\ M Cavassini ${ }^{7}$, R Weber $^{8}$, M Battegay ${ }^{9}, \mathrm{HC}$ Bucher $^{1,9}$ and the Swiss HIV Cohort Study \\ ${ }^{1}$ Basel Institute for Clinical Epidemiology and Biostatistics, University Hospital Basel, \\ Switzerland \\ 2 Internal Medicine, Kantonsspital Aarau, Switzerland \\ ${ }^{3}$ Regional Hospital of Lugano, Switzerland \\ ${ }^{4}$ Department of Infectious Diseases, University Hospital Bern and University of Bern, \\ Switzerland \\ ${ }^{5}$ Division of Infectious Diseases and Hospital Epidemiology, Cantonal Hospital St Gallen, \\ Switzerland \\ ${ }^{6}$ Division of Infectious Diseases, University Hospital Geneva, Switzerland \\ ${ }^{7}$ Division of Infectious Diseases, University Hospital Lausanne, Switzerland \\ ${ }^{8}$ Division of Infectious Diseases and Hospital Epidemiology, University Hospital Zurich, \\ University of Zurich, Switzerland \\ ${ }^{9}$ Division of Infectious Diseases and Hospital Epidemiology, University Hospital Basel, \\ Switzerland
}

"Corresponding author: Jim Young

Basel Institute for Clinical Epidemiology and Biostatistics, University Hospital Basel, CH-4031

Basel, Switzerland

Phone +41612653100

Fax: +41612653109

Email: james.young@usb.ch

Running head: Renal function after discontinuing tenofovir

Text 2003 words, 2 tables, 25 references

This study has been financed within the framework of the Swiss HIV Cohort Study, supported by the Swiss National Science Foundation (grant \# 33CS30_134277). The Basel Institute for Clinical Epidemiology and Biostatistics is supported by grants from Santésuisse and from the Gottfried and Julia Bangerter-Rhyner-Foundation. 


\begin{abstract}
Objectives: Tenofovir is associated with reduced renal function. It is not clear whether patients can be expected to fully recover their renal function if tenofovir is discontinued. Methods: We calculated the estimated glomerular filtration rate (eGFR) for patients in the Swiss HIV Cohort Study remaining on tenofovir for at least one year after starting a first antiretroviral therapy with tenofovir and either efavirenz or the ritonavir boosted protease inhibitors lopinavir, atazanavir or darunavir. We estimated the difference in eGFR slope between those who discontinued tenofovir after one year and those who remained on tenofovir.
\end{abstract}

Results: 1049 patients on tenofovir for at least one year were then followed for a median of 26 months, during which time 259 (25\%) patients discontinued tenofovir. After a year on tenofovir, the difference in eGFR between those starting with efavirenz and those starting with lopinavir , atazanavir and darunavir was -0.7 (95\% confidence interval $(\mathrm{Cl})-2.3$ to 0.8$),-1.4$ $(95 \% \mathrm{Cl}-3.2$ to 0.3$)$ and $0.0(95 \% \mathrm{Cl}-1.7$ to 1.70$) \mathrm{mL} / \mathrm{min}$ per $1.73 \mathrm{~m}^{2}$ respectively. The estimated linear rate of decline in eGFR on tenofovir was $-1.1(95 \% \mathrm{Cl}-1.5$ to -0.8$) \mathrm{mL} / \mathrm{min}$ per $1.73 \mathrm{~m}^{2}$ per year and its recovery after discontinuing tenofovir was 2.1 (95\% Cl 1.3 to 2.9$)$ $\mathrm{mL} / \mathrm{min}$ per $1.73 \mathrm{~m}^{2}$ per year. Patients starting tenofovir with either lopinavir or atazanavir appeared to have the same rates of decline and recovery as those starting tenofovir with efavirenz.

Conclusions: If patients discontinue tenofovir, clinicians can expect renal function to recover more rapidly than it declined.

Keywords: HIV, highly active antiretroviral therapy, kidney glomerulus, proximal kidney tubules 


\section{Introduction}

Tenofovir disoproxil fumarate (tenofovir) co-formulated with emtricitabine is the preferred reverse transcriptase inhibitor combination for patients with HIV when starting antiretroviral therapy [1]. Tenofovir is mainly eliminated by the kidneys and its use is associated with reduced renal function [2]. Regular monitoring of renal function is recommended for all patients on tenofovir [1].

While early reports suggest that renal function recovers completely when patients discontinue tenofovir $[3,4]$, larger recent studies suggest that some patients do not fully recover. In one study, only $42 \%$ of 24 patients recovered their pre-tenofovir estimated glomerular filtration rate (eGFR) [5]; in another, $59 \%$ of 183 patients returned to normal levels of eGFR but the time taken to recover varied considerably, with a mean of 22 months and an interquartile range (IQR) of 13 to 50 months [6].

It is difficult to estimate the rate of decline in eGFR for patients on tenofovir and its subsequent recovery if tenofovir is discontinued. First patients with lower eGFR are more likely to discontinue tenofovir [7]. Second the rate of recovery may well be greater in those patients who had a faster decline in eGFR [5]. These two factors create the potential for time dependent confounding, so that standard methods of analysis may lead to biased estimates [8].

In this study, we use marginal structural models to overcome the bias of standard methods. Using observational data from the Swiss HIV Cohort Study (SHCS), we estimate the rate of decline in eGFR for patients on tenofovir and the subsequent rate of recovery if tenofovir is then discontinued.

\section{Methods}

\section{Patients}

The SHCS is a multi-centre, prospective, observational cohort study with continuing enrolment of HIV-infected adults and routine follow up scheduled every 6 months [9]. In our study we included all patients starting their first antiretroviral therapy after 1 January 2002 , when routine measurement of serum creatinine measurements began in the SHCS. Patients had to start with tenofovir and either efavirenz (EFV) or the ritonavir boosted protease 
inhibitors (PI/r) lopinavir (LPV/r), atazanavir (ATV/r) or darunavir (DRV/r), plus either emtricitabine or lamivudine. Patients had to then remain on tenofovir for at least one year otherwise we could not expect any decline in eGFR nor subsequent recovery if the use of tenofovir then ceased - and have at least one calibrated serum creatinine measurement between 6 months and one year after starting therapy. We included in our analyses all measurements from the baseline at one year after starting therapy until the last recorded measurement to date or until patients re-started tenofovir having previously discontinued the drug, whichever came first. We calculated eGFR (in $\mathrm{ml} / \mathrm{min}$ per $1.73 \mathrm{~m} 2$ ) using the Chronic Kidney Disease Epidemiology Collaboration (CKD-EPI) formula [10]; we used only calibrated measurements because different SHCS centres use different measurement techniques.

\section{Statistical analyses}

We estimated the difference in eGFR over time between patients discontinuing tenofovir and patients remaining on tenofovir using marginal structural models for repeated measures $[8,11]$. These models used inverse probability weights for confounder control: treatment weights to adjust for differences in patients discontinuing or remaining on tenofovir over time; censoring weights to adjust for differences between patients continuing to provide measurements over time and those lost to follow up or re-starting tenofovir. Inverse probability weights were estimated by logistic regression using covariates likely to determine whether patients discontinued or re-started tenofovir or were lost to follow up: gender, ethnicity, injection drug use as the likely mode of transmission, age at baseline and initial antiretroviral regimen (EFV, LPV/r, ATV/r or DRV/r); and time-updated measurement of diabetes, hepatitis C co-infection, hypertension, body mass index, viral load, CD4 cell count and eGFR. We avoided extreme weights by truncating inverse probability weights at their 1st and 99th percentiles if below or above these values respectively [12].

In our main analysis, we fitted a sequence of three models. In Model 1, we used a cubic spline [13] to represent the decline in eGFR over time for patients remaining on tenofovir and a piecewise linear spline [14] to represent the difference in eGFR for those discontinuing tenofovir, with a change in slope at six months after discontinuation. In Model 2, we replaced the cubic spline with a straight line and in Model 3, we replaced both the cubic spline and piecewise linear spline with straight lines. 
In all three models, we assumed patients on different regimens might have different baseline values after one year on tenofovir but that the subsequent rate of decline and recovery before and after discontinuing tenofovir would be the same regardless of regimen. We did not have sufficient data to estimate differences in the rate of decline and recovery between therapies in a single model. Therefore in two exploratory analyses, we re-fitted this sequence of three models first to only those patients starting tenofovir with EFV and second to only those patients starting tenofovir with either LPV/r or ATV/r (as these two drugs appear to have similar effects on eGFR $[7,15]$ while little is known about the effect of $D R V / r$ ).

We used SAS version 9.2 (SAS Institute Inc., Cary, NC, USA) for all analyses.

\section{Results}

\section{Patient flow}

As at May 2013, 1049 patients had taken tenofovir continuously for at least one year, with at least one calibrated serum creatinine measurement between 6 months and one year after starting therapy (and having started one of the four regimens of interest - Table 1). These 1049 patients were then followed for a median of 26 months (IQR 13, 49). During this time, they had a median of 7 (IQR 3,12) creatinine measurements with a median time between measurements of 3.3 months (IQR 2.8, 5.6).

Of the 1049 patients, $888(85 \%)$ had a creatinine measurement in the 6 months prior to starting therapy. Patients started all four regimens with a similar median eGFR but after one year on therapy, the median eGFR was slightly lower in those starting tenofovir with a $\mathrm{Pl} / \mathrm{r}$ (Table 1). From baseline at one year, 259 (25\%) patients went on to discontinue tenofovir after an additional median time on tenofovir of 17 months (IQR 7, 34). Those starting therapy with LPV/r or ATV/r had a lower median eGFR when they discontinued tenofovir than those starting with EFV. Among the few patients $(45,4 \%)$ followed after discontinuing tenofovir for as long as they had been on tenofovir, eGFR returned to its pre-therapy median only in those patients that started tenofovir with EFV.

\section{Estimates of eGFR decline and recovery}

In Model 1, the estimated difference in eGFR slope between patients discontinuing tenofovir and patients remaining on tenofovir (Table 2) was not much greater in the first six months off 
tenofovir (4.1, 95\% confidence interval $(\mathrm{Cl})-1.0$ to $9.3\left[\mathrm{~mL} / \mathrm{min}\right.$ per $1.73 \mathrm{~m}^{2}$ per year]) than in the period beyond six months $(3.0,95 \% \mathrm{Cl} 1.8$ to 4.3$)$. This result suggests the difference was only slightly non-linear. These two estimates were not appreciable different when the flexible cubic spline representing the rate of decline in eGFR was replaced by a straight line (Model 2). This result suggests that after one year on tenofovir, further decline in eGFR was approximately linear. Finally, assuming recovery was also approximately linear (Model 3) provided estimates of an approximate linear rate of both decline in eGFR on tenofovir (-1.1, $95 \% \mathrm{Cl}-1.5$ to -0.8$)$ and its recovery after discontinuing tenofovir $(2.1,95 \% \mathrm{Cl} 1.3$ to 2.9$)$. In Model 1, patients starting tenofovir with DRV/r had similar eGFR at baseline to those starting tenofovir with EFV (estimated difference $0.0,95 \% \mathrm{Cl}-1.7$ to $1.7[\mathrm{~mL} / \mathrm{min}$ per 1.73 $\left.\mathrm{m}^{2}\right]$ ). There is some evidence patients starting tenofovir with LPV/r or ATV/r had a lower eGFR at baseline than those starting tenofovir with EFV (with estimated differences -0.7, 95\% $\mathrm{Cl}-2.3$ to 0.8 , and $-1.4,95 \% \mathrm{Cl}-3.2$ to 0.3 , respectively).

In the two exploratory analyses, estimates of the rate of decline in eGFR and its recovery after discontinuing tenofovir were similar when the three models were fit to either only those patients starting tenofovir with EFV or only those patients starting tenofovir with either LPV/r or ATV/r (Table 2).

\section{Discussion}

It is important to understand how eGFR recovers when tenofovir is discontinued because in the future, patients on tenofovir may switch to new treatment combinations [16]. Our modelling of observational data suggests that after one year on tenofovir, the approximate rate of further decline in eGFR is about $1 \mathrm{~mL} / \mathrm{min}$ per $1.73 \mathrm{~m}^{2}$ per year and is about half the approximate rate of recovery in eGFR if patients then discontinue taking tenofovir. This implies that most patients should recover pre-therapy renal function by the time they have been off tenofovir for as long as they were on it. Our data suggests this indeed happens, at least for patients starting tenofovir with EFV. For the 15 patients starting tenofovir with EFV and then remaining off tenofovir for as long as they were on it, the median difference between pre-therapy and post-recovery eGFR was 0.1 (IQR -9.4, 2.7) $\mathrm{mL} / \mathrm{min}$ per $1.73 \mathrm{~m}^{2}$.

For patients starting tenofovir with a $\mathrm{Pl} / \mathrm{r}$, the picture is more complicated. In an earlier study, we found an initial decrease in eGFR when starting tenofovir with LPV/r or ATV/r rather 
than with EFV, but no further decrease beyond the first six months of therapy [15]. And in this study, while patients starting tenofovir with LPV/r or ATV/r had lower eGFR at baseline (that is, after one year on tenofovir) than patients starting tenofovir with EFV, both groups of patients had a similar rate of decline in eGFR beyond one year and a similar rate of recovery when tenofovir was discontinued.

However patients starting tenofovir with a $\mathrm{Pl} / \mathrm{r}$ did not appear to recover their pretherapy eGFR. For the 30 patients starting tenofovir with a $\mathrm{Pl} / \mathrm{r}$ and then remaining off tenofovir for as long as they were on it, the median difference between pre-therapy and postrecovery eGFR was $-7.9(\mathrm{IQR}-15.9,3.6) \mathrm{mL} / \mathrm{min}$ per $1.73 \mathrm{~m}^{2}$. Of these 30 patients, 18 were still on a $\mathrm{Pl} / \mathrm{r}$ post-recovery. Ritonavir inhibits creatinine secretion [17] and the corresponding immediate but non-progressive increase in serum creatinine leads to a one-off decrease in eGFR [18]. Hence patients on tenofovir with LPV/r or ATV/r might still recover their true pretherapy glomerular filtration rate after discontinuing tenofovir without recovering their pretherapy eGFR. Analyses using eGFR must be interpreted with caution $[18,19]:$ LPV/r and ATV/r are thought to increase the risk of renal impairment [7] but their contribution could be exaggerated when eGFR is used to assess that risk.

Note that our estimated rate of decline in eGFR after one year is the same as the accepted "normal" decline in glomerular filtration rate with age $\left(1 \mathrm{~mL} / \mathrm{min}\right.$ per $1.73 \mathrm{~m}^{2}$ per year [20]). However much of this "normal" decline has been attributed to comorbidities that inevitably accompany ageing [21-23]. This makes it difficult to judge how much tenofovir has contributed to the rate of decline in renal function in these patients. That the decline in eGFR reversed when tenofovir was discontinued suggests that tenofovir was responsible, at least in part, for the decline.

This study has the same limitations as our earlier study [15]. In addition, we did not have sufficient data to estimate, in a single model, differences between regimens in the rate of decline in eGFR and its recovery if tenofovir is discontinued. This can be done using a marginal structural model but requires artificial censoring of patients when they change their baseline regimen $[24,25]$ and this artificial censoring reduces the power of the analysis. The strengths of this study are the use of calibrated creatinine measurements and of statistical methods that minimise any bias due to time dependent confounding. 
When monitoring eGFR in patients starting tenofovir with either EFV, LPV/r or ATV/r, clinicians can expect renal function to recover more rapidly than it declined if patients discontinue tenofovir. On average patients on a $\mathrm{Pl} / \mathrm{r}$ may not recover their pre-therapy eGFR after discontinuing tenofovir, but is not clear yet whether this has clinical consequences. While on average patients can be expected to recover their renal function after discontinuing tenofovir, not all patients will do so.

\section{Acknowledgements}

This study has been financed within the framework of the Swiss HIV Cohort Study, supported by the Swiss National Science Foundation (grant \# 134277). The data are gathered in 7 outpatient clinics, 15 regional hospitals and by 36 practitioners. The Basel Institute for Clinical Epidemiology and Biostatistics is supported by grants from Santésuisse and from the Gottfried and Julia Bangerter-Rhyner-Foundation.

The members of the Swiss HIV Cohort Study are Aubert V, Barth J, Battegay M, Bernasconi E, Böni J, Bucher HC, Burton-Jeangros C, Calmy A, Cavassini M, Egger M, Elzi L, Fehr J, Fellay J, Furrer H (Chairman of the Clinical and Laboratory Committee), Fux CA, Gorgievski M, Günthard H (President of the SHCS), Haerry D (deputy of "Positive Council"), Hasse B, Hirsch HH, Hösli I, Kahlert C, Kaiser L, Keiser O, Klimkait T, Kouyos R, Kovari H, Ledergerber B, Martinetti G, Martinez de Tejada B, Metzner K, Müller N, Nadal D, Pantaleo G, Rauch A (Chairman of the Scientific Board), Regenass S, Rickenbach M (Head of Data Center), Rudin C (Chairman of the Mother \& Child Substudy), Schöni-Affolter F, Schmid P, Schultze D, Schüpbach J, Speck R, Staehelin C, Tarr P, Telenti A, Trkola A, Vernazza P, Weber R, Yerly S.

Author's contributions: $\mathrm{CF}, \mathrm{EB}, \mathrm{HF}, \mathrm{PV}, \mathrm{AC}, \mathrm{MC}, \mathrm{RW}, \mathrm{MB}$ were responsible for data collection in their respective hospitals; HB, JY and QW designed this study; QW and JY carried out the statistical analyses; JY and QW wrote the first draft of the manuscript; JY, QW, and HB revised the manuscript; all authors reviewed, commented on, and approved the final version of the manuscript.

The following authors have received honoraria, travel grants or unrestricted research grants from Abbott, Bristol-Meyers Squibb, Gilead or Tibotec: CF, EB, HF, PV, RW, MB, HB. 


\section{References}

1 Panel on Antiretroviral Guidelines for Adults and Adolescents. Guidelines for the use of antiretroviral agents in HIV-1-infected adults and adolescents. Department of Health and Human Services. 12 February 2013. Available at http://aidsinfo.nih.gov/contentfiles/AdultandAdolescentGL.pdf (accessed 3 October 2013).

2 Cooper RD, Wiebe N, Smith N, Keiser P, Naicker S, Tonelli M. Systematic review and meta-analysis: renal safety of tenofovir disoproxil fumarate in HIV-infected patients. Clin Infect Dis 2010; 51: 496-505.

3 Izzedine $\mathrm{H}$, Isnard-Bagnis C, Hulot JS et al. Renal safety of tenofovir in HIV treatmentexperienced patients. AIDS 2004; 18: 1074-1076.

4 Herlitz LC, Mohan S, Stokes MB, Radhakrishnan J, D'Agati VD, Markowitz GS. Tenofovir nephrotoxicity: acute tubular necrosis with distinctive clinical, pathological, and mitochondrial abnormalities. Kidney Int 2010; 78: 1171-1177.

5 Wever K, van Agtmael MA, Carr A. Incomplete reversibility of tenofovir-related renal toxicity in HIV-infected men. J Acquir Immune Defic Syndr 2010; 55: 78-81.

6 Bonjoch A, Echeverria P, Perez-Alvarez $\mathrm{N}$ et al. High rate of reversibility of renal damage in a cohort of HIV-infected patients receiving tenofovir-containing antiretroviral therapy. Antiviral Res 2012; 96: 65-69.

7 Ryom L, Mocroft A, Kirk O et al. Association between antiretroviral exposure and renal impairment among HIV-positive persons with normal baseline renal function: the D:A:D study. J Infect Dis 2013; 207: 1359-1369.

8 Hernan MA, Brumback BA, Robins JM. Estimating the causal effect of zidovudine on CD4 count with a marginal structural model for repeated measures. Stat Med 2002; 21: 16891709.

9 Schoeni-Affolter F, Ledergerber B, Rickenbach M et al. Cohort profile: the Swiss HIV Cohort study. Int J Epidemiol 2010; 39: 1179-1189. 
10 Levey AS, Stevens LA, Schmid CH et al. A new equation to estimate glomerular filtration rate. Ann Intern Med 2009; 150: 604-612.

11 Cole SR, Hernan MA, Margolick JB, Cohen MH, Robins JM. Marginal structural models for estimating the effect of highly active antiretroviral therapy initiation on CD4 cell count. Am J Epidemiol 2005; 162: 471-478.

12 Cole SR, Hernan MA. Constructing inverse probability weights for marginal structural models. Am J Epidemiol 2008; 168: 656-664.

13 Hernan MA, Brumback B, Robins JM. Marginal structural models to estimate the causal effect of zidovudine on the survival of HIV-positive men. Epidemiology 2000; 11: 561-570.

14 Greenland S. Dose-response and trend analysis in epidemiology: alternatives to categorical analysis. Epidemiology 1995; 6: 356-365.

15 Young J, Schafer J, Fux CA et al. Renal function in patients with HIV starting therapy with tenofovir and either efavirenz, lopinavir or atazanavir. AIDS 2012; 26: 567-575.

16 Walmsley SL, Antela A, Clumeck $\mathrm{N}$ et al. Dolutegravir plus abacavir-lamivudine for the treatment of HIV-1 infection. N Engl J Med 2013; 369: 1807-1818.

17 Lepist El, Murray BP, Tong L, Roy A, Bannister R, Ray AS. Effect of cobicistat and ritonavir on proximal renal tubular cell uptake and efflux transporters. 51st Interscience Conference on Antimicrobial Agents and Chemotherapy. 17-20 September 2011, Chicago, USA. Abstract A1-1724.

18 Gallant JE, Koenig E, Andrade-Villanueva J et al. Cobicistat versus ritonavir as a pharmacoenhancer of atazanavir plus emtricitabine/tenofovir disoproxil fumarate in treatment-naive HIV type 1-infected patients: week 48 results. J Infect Dis 2013; 208: 3239.

19 Fux CA, Simcock M, Wolbers M et al. Tenofovir use is associated with a reduction in calculated glomerular filtration rates in the Swiss HIV Cohort Study. Antivir Ther 2007; 12 : 1165-1173. 
$20 \mathrm{~K} / \mathrm{DOQ}$ clinical practice guidelines for chronic kidney disease: evaluation, classification, and stratification. Am J Kidney Dis 2002; 39: S1-266.

21 Lindeman RD, Tobin J, Shock NW. Longitudinal studies on the rate of decline in renal function with age. J Am Geriatr Soc 1985; 33: 278-285.

22 Fliser D, Franek E, Ritz E. Renal function in the elderly--is the dogma of an inexorable decline of renal function correct? Nephrol Dial Transplant 1997; 12: 1553-1555.

23 Lindeman RD. Is the decline in renal function with normal aging inevitable? Geriatr Nephrol Urol 1998; 8: 7-9.

24 Robins JM, Hernan MA, Brumback B. Marginal structural models and causal inference in epidemiology. Epidemiology 2000; 11: 550-560.

25 Hernan MA, Lanoy E, Costagliola D, Robins JM. Comparison of dynamic treatment regimes via inverse probability weighting. Basic Clin Pharmacol Toxicol 2006; 98: 237242. 
Table 1: A: Patient characteristics at one year after starting tenofovir (TDF) with either efavirenz (EFV) or the ritonavir-boosted protease inhibitors lopinavir (LPV/r), atazanavir (ATV/r) or darunavir (DRV/r), plus either emtricitabine or lamivudine. B: Patient flow before and after starting antiretroviral therapy and at each point, the corresponding median estimated glomerular filtration rate (eGFR) and interquartile range (IQR).

\begin{tabular}{|c|c|c|c|c|}
\hline A: Characteristics & $\begin{array}{l}\mathrm{EFV} \\
\mathrm{n}=501\end{array}$ & $\begin{array}{l}\mathrm{LPV} / \mathrm{r} \\
\mathrm{n}=219\end{array}$ & $\begin{array}{l}\text { ATV/r } \\
n=189\end{array}$ & $\begin{array}{l}\mathrm{DRV} / \mathrm{r} \\
\mathrm{n}=140\end{array}$ \\
\hline Median age, years & 41 & 42 & 40 & 41 \\
\hline Female gender, \% & 16 & 25 & 23 & 10 \\
\hline Black ethnicity, \% & 15 & 13 & 11 & 6 \\
\hline $\begin{array}{l}\text { Intravenous drug use as the likely } \\
\text { mode of transmission, } \%\end{array}$ & 5 & 10 & 13 & 3 \\
\hline $\begin{array}{l}\text { Median time since HIV diagnosis, } \\
\text { months }\end{array}$ & 23 & 15 & 32 & 14 \\
\hline Advance infection (CDC Group C), \% & 14 & 30 & 14 & 18 \\
\hline Hepatitis C co-infection, \% & 10 & 16 & 21 & 6 \\
\hline Diabetes, \% & 5 & 3 & 3 & 1 \\
\hline Hypertension, \% & 23 & 27 & 22 & 26 \\
\hline Median HIV RNA, $\log _{10}$ copies/ml & 1.3 & 1.6 & 1.5 & 1.3 \\
\hline Median CD4 cell count, cells $/ \mathrm{mm}^{3}$ & 421 & 412 & 428 & 465 \\
\hline Median body mass index, $\mathrm{kg} / \mathrm{m}^{2}$ & 24 & 23 & 24 & 24 \\
\hline \multicolumn{5}{|l|}{ B: Patient flow } \\
\hline \multicolumn{5}{|c|}{ Number of patients with eGFR measurements } \\
\hline Prior to starting TDF & 417 & 174 & 166 & 131 \\
\hline One year after starting TDF & 501 & 219 & 189 & 140 \\
\hline Prior to stopping TDF & 103 & 77 & 63 & 16 \\
\hline $\begin{array}{l}\text { Remaining off TDF for as long as } \\
\text { having received TDF }\end{array}$ & 15 & 18 & 11 & 1 \\
\hline \multicolumn{5}{|l|}{ Median eGFR, mL/min per $1.73 \mathrm{~m}^{2}$ (IQR) } \\
\hline Prior to starting TDF & $105(93,115)$ & $106(95,115)$ & $107(95,116)$ & $107(94,118)$ \\
\hline One year after starting TDF & $104(94,115)$ & $102(87,111)$ & $100(85,110)$ & $100(89,111)$ \\
\hline Prior to stopping TDF & $99(83,114)$ & $94(73,109)$ & $94(76,106)$ & $103(69,112)$ \\
\hline $\begin{array}{l}\text { Remaining off TDF for as long as } \\
\text { having received TDF }\end{array}$ & $104(71,114)$ & $95(80,110)$ & $82(73,103)$ & $84(84,84)$ \\
\hline
\end{tabular}


Table 2: Estimates of the decline and recovery in estimated glomerular filtration rate (eGFR) for patients starting tenofovir (TDF) with either efavirenz (EFV) or the ritonavir boosted protease inhibitors lopinavir (LPV/r), atazanavir (ATV/r) or darunavir, plus either emtricitabine or lamivudine.

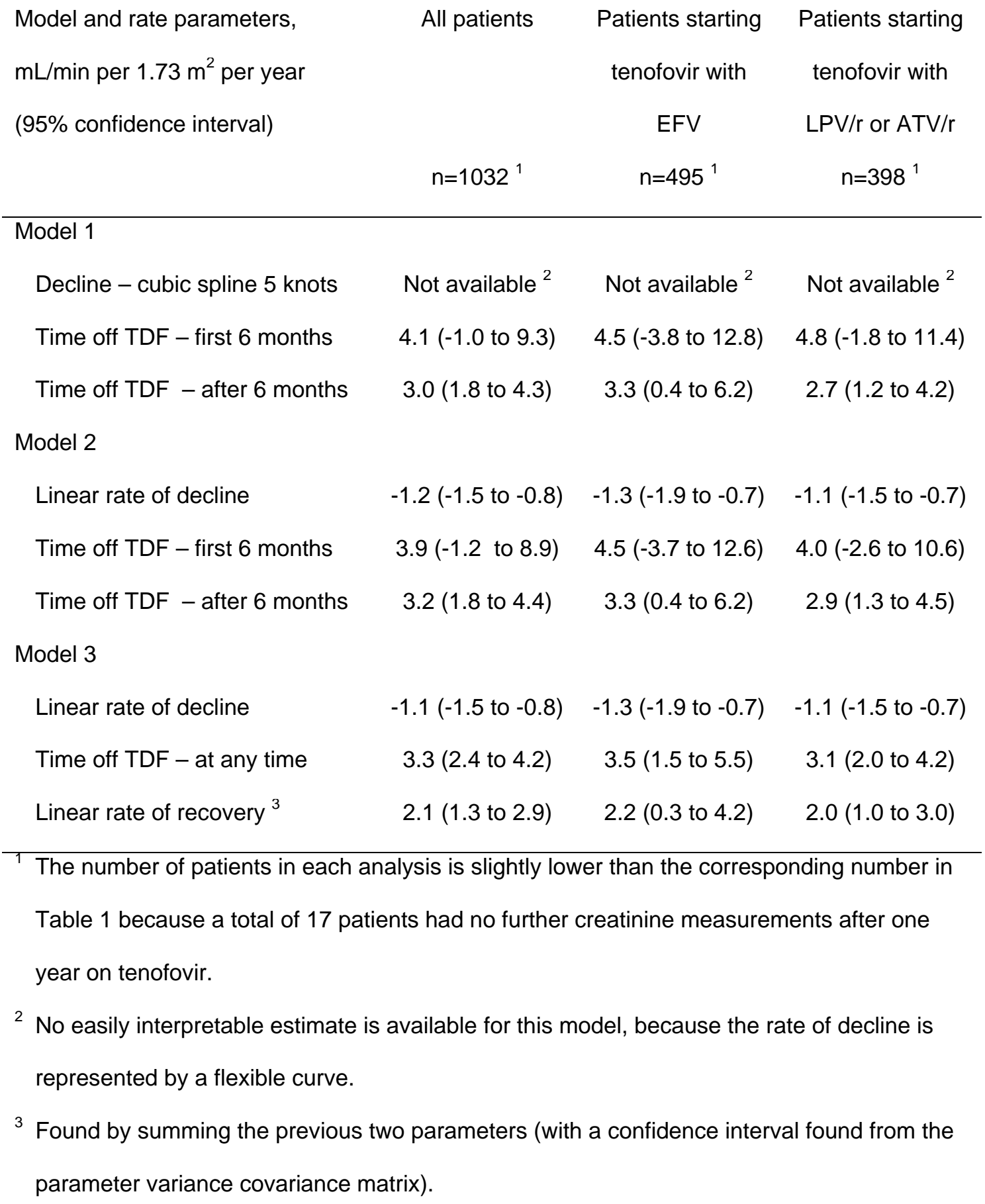

